\title{
PROCESOS COGNITIVOS Y ESTRATEGIAS PSICOLINGÜÍSTICAS QUE INTERVIENEN EN LA LECTURA COMPRENSIVA: DISEÑO Y EJECUCIÓN DE UN PROGRAMA EXPERIMENTAL EN NIÑOS CON PROBLEMAS DE APRENDIZAJE
}

\author{
Ricardo Canales G. ${ }^{1}$ \\ Universidad Nacional Mayor de San Marcos, Lima-Perú \\ (RECIBIDO 5/01/2008, ACEPTADO 25/04/2008)
}

\begin{abstract}
RESUMEN
Se trata de una investigación que presenta los hallazgos encontrados en el campo de la Comprensión Lectora. Partiendo del modelo teórico de la psicología cognitiva y la psicolingüística, y en particular de la teoría de Van Dijk y Kintsch $(1983,1993,1994)$, el autor explora la problemática de la lectura comprensiva, en una muestra de niños y adolescentes con problemas de aprendizaje de la provincia del Callao, tratando de conocer hasta qué punto se puede intervenir en la mejora de sus deficiencias lectoras, para ello se diseña y aplica un Programa Experimental de Tratamiento.
\end{abstract}

Como hallazgo fundamental del estudio, se encontró, luego de aplicado el Programa, diferencia significativa en los niveles de comprensión lectora alcanzados en el pre test y el post test, por los alumnos del agrupamiento I (secundaria) expuestos al programa experimental; así como la mejoría en los alumnos del agrupamiento II (primaria).

Palabras clave: Comprensión lectora, Problemas de aprendizaje, Dislexia, Retardo lector, Estrategias de comprensión lectora, Programa Experimental de Tratamiento.

\begin{abstract}
The investigation shows the results founded in the field of Reading Comprehension. Starting from the theoretic model of cognitive psychology and psycholinguistics, and in particular the theory of Van Dijk and Kintsch (1983, 1993, 1994). The author explores the problematic of comprehensive reading in a group of children and teenagers with learning disabilities in the province of Callao, trying to know how much can they could intervening in the improvement of their reading weakness, for what we have design and application and an Experimental Program of Treatment.

The most important idea of the study was the significant difference in reading comprehension levels reached in the pre test and in the post test, by the group I students after applying the experimental program, and as an important improvement in the student group II.
\end{abstract}

Keywords: Reading comprehension, Learning disabilities, Dislexy, Reading retardation, Strategies of reading comprehension, Experimental.

1 Docente de la Facultad de Psicología de la Universidad Nacional Mayor de San Marcos, Lima-Perú. 


\section{INTRODUCCIÓN}

En el Perú, tenemos un grave problema con la lectura. Hace unos años se publicó en un Boletín del Ministerio de Educación (2001) los resultados de un estudio internacional realizado por el Laboratorio de Medición de la Calidad financiado por el BID. Se realizó un análisis comparativo sobre los resultados de la educación básica entre los países de la región; participaron Argentina, Bolivia, Brasil, Chile, Colombia, Costa Rica, Cuba, Honduras, México, Paraguay, Perú, República Dominicana y Venezuela. Se aplicaron más de 54 mil pruebas de matemáticas y lenguaje en tercer y cuarto grado de todos estos países (sobre 18 millones en total) entre junio y noviembre. Los alumnos del Perú quedaron ubicados en último lugar en Matemáticas y antepenúltimo lugar en Lenguaje.

Años más tarde, en la evaluación mundial de la prueba PISA (2003) sobre competencias lectoras (decodificación, comprensión y juicio crítico), llevada a cabo por la UNESCO, los adolescentes peruanos, lamentablemente, ocuparon el último lugar entre 43 países del mundo.

En nuestro país en los últimos tiempos se han realizado aproximaciones a dicha problemática. Tapia (1995) realizó una Investigación de Evaluación Formativa sobre las bondades de un Programa de Desarrollo de la Comprensión Lectora en niños Deficientes Lectores de 4to y 5to grado de Nivel Socioeconómico Bajo. Este Programa se basó en la Aplicación de Estrategias Cognitivas y Metacognitivas para el mejoramiento de la Comprensión Lectora en niños deficientes lectores. Los resultados demostraron que los niños deficientes lectores no solo superaron el déficit encontrado al inicio de la aplicación del Programa, sino que incluso superaron significativamente al grupo de buenos lectores. Pinzás (1997) examina los efectos de la enseñanza de habilidades metacognitivas sobre la comprensión lectora. En una muestra total de 1553 niños, en donde su usó grupo control y aplicaron los procedimientos estadísticos de rigor, se encontró que el entrenamiento en este tipo de habilidades era más efectivo que el entrenamiento en otras. La conciencia metacognitiva y la ejecución de acciones de monitoreo se vio mejorada con el uso de la técnica de provocar las disonancias cognitivas. Velarde, E. (2001) encontró que en niños que cursaban el tercer grado de primaria en el Cercado del Callao, se daba una relación entre los niveles de conciencia fonológica y la lectura (decodificación y comprensión). Uno de los últimos estudios de Gonzáles, R. (1998) resulta por demás preocupante; en él se señala que habiendo examinado a 311 sujetos ingresantes a dos universidades: una particular y otra estatal, ninguno llegó al nivel independiente de comprensión lectora, sólo 6 sujetos alcanzaron un nivel dependiente instruccional, y 305 sujetos presentaban un nivel deficitario, es decir tenían dificultades muy serias en la comprensión lectora.

\section{Problema}

Es a partir de esta preocupación que se realiza la presente investigación, la cual tiene como propósito ir dando cuenta de los procesos cognitivos y psicolingüísticos que subyacen a la comprensión lectora -contribuyendo de este modo a la estructuración de un modelo teórico más comprensivo sobre dicha competencia-, así como diseñar un programa experimental de tratamiento para mejorar la comprensión lectora en niños y adolescentes con problemas de aprendizaje en nuestro medio. 
Para efectivizar el presente estudio, se escogió una muestra de niños con problemas de aprendizaje de un colegio particular del Callao, la mayoría de los cuales provenían de hogares de nivel socioeconómico medio y medio bajo.

El conjunto de todas estas consideraciones llevó a tratar de buscar respuestas a las siguientes interrogantes:

- ¿Cuáles son los niveles de comprensión lectora que presentan los estudiantes con problemas de aprendizaje del $3^{\circ}$ al $5^{\circ}$ de primaria, y los alumnos del $1^{\circ}$ al $2^{\circ}$ de secundaria?

- ¿Qué procesos cognitivos y psicolingüísticos subyacen a sus niveles de comprensión lectora?

- ¿Qué importancia tendrán la inteligencia verbal, los procesos cognitivos, como memoria e inferencias, y los procesos psicolingüísticos, como los sintácticossemánticos, en las tareas de comprensión lectora?

- ¿Es factible mejorar sus niveles de comprensión lectora aplicando un Programa Experimental de Tratamiento (PET)?

- ¿Existirán diferencias significativas entre los niveles de comprensión lectora y las funciones psicolingüísticas sintácticas-semánticas, entre los niños sometidos al tratamiento y los niños que no lo recibieron?

- ¿Qué modelo teórico puede explicar mejor la complejidad de los procesos cognitivos y psicolingüísticos intervinientes en la comprensión lectora?

\section{Objetivos}

\section{General}

Formular y evaluar los efectos de un Programa Experimental para el mejoramiento de la comprensión lectora en alumnos con problemas de aprendizaje de $3^{\circ}, 4^{\circ}$ y $5^{\circ}$ de primaria; así como de $1^{\circ}$ y $2^{\circ}$ de secundaria de la zona urbana del Callao.

\section{Específicos}

1. Evaluar y comparar los niveles de comprensión lectora y los procesos sintácticossemánticos en los niños y adolescentes de la muestra total.

2. Evaluar comparar los niveles de comprensión lectora y los procesos sintácticossemánticos en los alumnos de $1 .^{\circ}-5 .^{\circ}$ primaria, y de $1 .^{\circ}-2 .^{\circ}$ secundaria del Grupo Experimental (pre y post test).

3. Evaluar y comparar la inteligencia verbal y los niveles de comprensión lectora en los niños y adolescentes de la muestra total.

4. Evaluar y comparar los niveles de comprensión lectora y la memoria verbal de corto plazo en los niños y adolescentes de la muestra. 
5. Comparar los grados de avance en los procesos sintácticos-semánticos de los alumnos sometidos al tratamiento: Grupo Experimental y los procesos sintácticos-semánticos de los que no lo recibieron: Grupo Control.

6. Evaluar los efectos del Programa experimental de tratamiento y aportar en la elaboración de un modelo teórico que explique mejor la complejidad de los procesos intervinientes en la comprensión lectora.

\section{Modelo teórico}

Es un Modelo de representación semántica: el significado del texto se representa como una red de proposiciones, donde la información nueva se integra en una red proposicional.

Operan 3 niveles de procesamiento:

I) El nivel microestructural.

II) El nivel macroestructural.

III) El nivel superestructural.

\section{Supuestos básicos}

a) Un punto de vista psicolingüístico acerca de los problemas de aprendizaje de la lectura: Partiendo de los estudios, investigaciones y experimentos cruciales de Vellutino et al. (1972, 1979, 1987, 1995), y los que continuaron su línea de investigación, se elaboró un MODELO EXPLICATIVO SOBRE LA DISLEXIA, considerándolo como producto de una deficiencia específica del LENGUAJE que impide al sujeto verbalizar la información ortográfica. Esto produciría fallas en la decodificación y luego en la comprensión lectora.

La incapacidad para verbalizar la información ortográfica se debe principalmente, según Vellutino, a un déficit específico en la MEMORIa VERBaL operacional de CORTo PLAzo, que es la que almacena la secuencia auditiva-fonémica, permitiendo integrar la información y emitir correctamente una palabra, la cual a su vez contiene un significado verbal.

Para Vellutino y sus colaboradores, la dislexia es, entonces, causada por deficiencias en la codificación lingüística y no por los procesos perceptivo visuales o secuenciales.

b) Un punto de vista constructivista e interactivo sobre la comprensión lectora: Van Dijk y Kintsch sostienen que la comprensión lectora supone la CONSTRUCCIÓN DE UN MODELO MENTAL Y SITUACIONAL que dé cuenta de aquello que el autor nos quiere transmitir; en tal sentido, podemos entender a la comprensión como el resultado del encuentro de lo que contiene el texto en sí y una mente que lee.

En este enfoque quizá lo más relevante viene a ser el acento que se pone en el papel que juegan los conocimientos previos que el sujeto trae. 
En el proceso de construir la representación mental respecto al texto, se ponen en juego diversas estrategias, unas son de carácter sintáctico, otras de carácter semántico y otras de proyección semántica o de construcción de la macroestructura, que vienen a ser las macrorreglas o estrategias de borrado, generalización y elaboración.

En esencia, comprender un texto implica haber arribado a la macroestructura, es decir haber captado la idea principal, la tesis central que el autor ha planteado.

c) Intervención para el mejoramiento de la comprensión lectora: A partir de las investigaciones de García Madruga, Cordero, Luque y Santa María (1995) y García Madruga, Elosúa, Luque, Gutiérrez y Gárate (1999), se comprueba el éxito de los programas de intervención en el mejoramiento de la comprensión lectora. Aquí lo esencial no sólo consiste en que el sujeto aprenda las estrategias básicas de comprensión lectora (supresión, generalización y elaboración) sino que pueda practicarlas constantemente.

\section{Variables de estudio}

Variable independiente: Programa Experimental para mejorar la comprensión lectora.

Variable dependiente: El Nivel de comprensión lectora de los alumnos expuestos al programa, diferenciados por grados y agrupamientos, y medido a través del Test de Condemarín: CLP y Test de Cloze (habilidades sintácticas-semánticas).

\section{Hipótesis}

\section{General}

El nivel de Comprensión Lectora de los alumnos con problemas de aprendizaje, expuestos al Programa Experimental, se verá incrementado en mayor medida que el nivel de Comprensión Lectora de los alumnos con problemas de aprendizaje no sometidos al Programa Experimental, tanto en el agrupamiento I $\left(3 .^{\circ}, 4 .^{\circ}\right.$ y $5 .^{\circ}$ de primaria) como en el agrupamiento II $\left(1 .^{\circ}\right.$ y $2 .^{\circ}$ de secundaria).

\section{Hipótesis de trabajo}

Hipótesis N. ${ }^{0} 1$

Hipótesis Nula Ho: "No existe diferencia significativa entre los niveles de Comprensión Lectora alcanzados en el pre test y el post test, por los alumnos con problemas de aprendizaje expuestos al Programa Experimental del Agrupamiento I (alumnos de $3 .^{\circ}$ a $5 .{ }^{\circ}$ de primaria)".

Hipótesis Alterna H1:"Existe diferencia significativa entre los niveles de Comprensión Lectora alcanzados en el pre test y el post test, por los alumnos con problemas de aprendizaje expuestos al Programa Experimental del Agrupamiento I (alumnos de $3 .^{\circ}$ a $5 .{ }^{\circ}$ de primaria)". 


\section{Hipótesis $N^{\circ} 2$}

Hipótesis Nula Ho: "No existe diferencia significativa entre los niveles de Comprensión Lectora alcanzados en el pre test y el post test, por los alumnos con problemas de aprendizaje expuestos al Programa Experimental del Agrupamiento II (alumnos de $1 .^{\circ} \mathrm{y}$ $2 .^{\circ}$ de secundaria)".

Hipótesis Alterna H2: "Existe diferencia significativa entre los niveles de Comprensión Lectora alcanzados en el pre test y el post test, por los alumnos con problemas de aprendizaje no expuestos al Programa Experimental del Agrupamiento II (alumnos de $1 .^{\circ}$ y $2 .^{\circ}$ de secundaria)".

\section{Hipótesis $N^{\circ} 3$}

Hipótesis Nula Ho: "Los niños y adolescentes de los Agrupamientos I y II, diferenciados por grados escolares y niveles de lectura, no lograrán avances importantes, luego de ser expuestos al Programa Experimental".

Hipótesis Alterna H3: "Los niños y adolescentes de los Agrupamientos I y II, diferenciados por grados escolares y niveles de lectura, lograrán avances importantes, luego de ser expuestos al Programa Experimental".

Hipótesis secundarias:

1. Existe correlación positiva entre las habilidades sintácticas-semánticas y la comprensión lectora en los sujetos de la muestra.

2. Existe correlación positiva entre las habilidades sintácticas-semánticas y la comprensión lectora en los niños con problemas de aprendizaje del Grupo Experimental - Pre Test.

3. Existe correlación positiva entre las habilidades sintácticas-semánticas y la comprensión lectora en los niños con problemas de aprendizaje expuestos al Programa Experimental - Post Test.

4. Existe correlación positiva entre el nivel de Inteligencia verbal y los procesos psicolingüísticos sintácticos-semánticos en los niños con problemas de aprendizaje de la muestra.

5. Existe correlación positiva entre el nivel de memoria verbal de corto plazo y la comprensión lectora en los niños con problemas de aprendizaje de la muestra.

6. Los procesos psicolingüísticos sintácticos-semánticos de los niños con problemas de aprendizaje expuestos al Programa Experimental se verán incrementados en mayor grado que los procesos psicolingüísticos sintácticos-semánticos de los niños con problemas de aprendizaje no expuestos al Programa Experimental.

\section{MÉTODO}

La presente es una investigación de tipo sustantiva-explicativa, por cuanto se orientó a describir, comparar y descubrir algunos factores que incidirían en el aprendizaje de la lectura comprensiva, con diseño experimental: 


$\begin{array}{lllll}\text { Grupo Exp. } & \text { Aleat. } & \text { O1 } & \text { X } & \text { O2 } \\ \text { Grupo Contr. } & \text { Aleat. } & \text { O3 } & & \text { O4 }\end{array}$

\section{Población y muestra}

La Población estuvo constituida por los niños del nivel socioeconómico medio bajo de la Provincia Constitucional del Callao, que presentan problemas del aprendizaje en la lectura.

La Muestra fue no probabilística, de carácter intencional, que consideró la edad y el grado escolar, así como la pertenencia al grupo de niños con problemas de aprendizaje. Total: 46 sujetos.

Tabla N. ${ }^{0}$ 1. Distribución de la muestra de los sujetos del grupo experimental y grupo control por niveles y género.

\begin{tabular}{lccccc}
\hline \multicolumn{1}{c}{ Sujetos } & \multicolumn{2}{c}{ Agrupamiento I } & \multicolumn{2}{c}{ Agrupamiento II } & $\mathbf{N .}^{\mathbf{0}}$ de sujetos \\
\hline & $\begin{array}{c}\text { Grupo } \\
\text { Experimental }\end{array}$ & $\begin{array}{c}\text { Grupo } \\
\text { Control }\end{array}$ & $\begin{array}{c}\text { Grupo } \\
\text { Experimental }\end{array}$ & $\begin{array}{c}\text { Grupo } \\
\text { Control }\end{array}$ & \\
\hline Masculino & 11 & 13 & 6 & 8 & 38 \\
\hline Femenino & 2 & 0 & 4 & 2 & 8 \\
\hline Total & $\mathbf{1 3}$ & $\mathbf{1 3}$ & $\mathbf{1 0}$ & $\mathbf{1 0}$ & $\mathbf{4 6}$ \\
\hline
\end{tabular}

\section{Instrumentos}

1. Test de inteligencia de la escala Wechsler (Wisc-R).

2. Prueba de comprensión lectora CLP de Condemarín, Allende y Milicic, forma A (pre test) y forma B (post test).

3. Test Cloze.

4. Programa de tratamiento experimental dirigido a mejorar la comprensión lectora.

\section{RESULTADOS}

El objetivo fundamental de la presente investigación, coherente con nuestra hipótesis general, fue comprobar si se producían diferencias significativas a nivel de compresión lectora entre los alumnos expuestos al programa experimental y los que no lo estuvieron. Para ello, se trabajó con una muestra elegida con criterio no probabilístico, de carácter intencional, que consideró la edad y el grado escolar, así como la pertenencia al grupo de niños con problemas de aprendizaje en un colegio especializado del Callao, perteneciente al nivel socioeconómico medio bajo. En tal sentido, se quiso ver si existen diferencias entre los puntajes medios alcanzados por el grupo experimental y el grupo control, en la variable dependiente: nivel de comprensión lectora. 
En los Cuadros N. ${ }^{\circ} 1$ y 2 se muestran datos estadísticos descriptivos (medias de grados escolares y de agrupamientos), así como los valores " $\mathrm{t}$ " resultantes de las comparaciones entre las medias, en el Grupo Experimental y en Grupo Control.

Cuadro ${ }^{\circ}{ }^{\circ}$ 1. Medias de grupo experimental en CLP.

\begin{tabular}{|c|c|c|c|c|c|c|c|c|c|c|c|}
\hline & & & $\begin{array}{c}\text { PRE } \\
\text { TEST }\end{array}$ & & & & $\begin{array}{l}\text { POST } \\
\text { TEST }\end{array}$ & & GL & $\mathrm{t}$ & \\
\hline & Grado & N. ${ }^{o}$ & Media & Percentil & $\mathrm{T}$ & Media & Percentil & $\mathrm{T}$ & & & \\
\hline Agrupam. & $3^{\circ}$ prim. & 3 & 15.33 & 20 & 37 & 17.66 & 36 & 44.9 & & & \\
\hline \multirow[t]{3}{*}{ Primaria } & $4^{\circ}$ prim. & 6 & 10.83 & 17.77 & 38.2 & 12.16 & 34.44 & 41.85 & & & \\
\hline & $5^{\circ}$ prim. & 4 & 7.5 & 17.5 & 39.05 & 6.75 & 15 & 38.92 & & & \\
\hline & & & 11.2 & 18.42 & 38.08 & 12.19 & 28.48 & 41.89 & 12 & $\mathrm{t}=0.685$ & No hay dif. \\
\hline Agrupam. & $1^{\circ} \mathrm{sec}$. & 4 & 8.25 & 15 & 36.02 & 10 & 22.5 & 38.92 & & & \\
\hline \multirow[t]{4}{*}{ Secundaria } & $2^{\circ} \mathrm{sec}$. & 6 & 15.16 & 26.66 & 40.18 & 18.83 & 35 & 44.93 & & & \\
\hline & & & 23.41 & 41.66 & 76.2 & 28.83 & 57.5 & 83.85 & & & \\
\hline & & & 11.7 & 20.83 & 38.1 & 14.41 & 28.75 & 41.92 & 9 & $\mathrm{t}=2.225$ & *Hay dif. Sign. \\
\hline & $* \mathrm{P}<0.05$ & & & & & & & & & & \\
\hline
\end{tabular}

Cuadro N. ${ }^{\circ}$ 2. Medias de grupo control en CLP.

\begin{tabular}{|c|c|c|c|c|c|c|c|c|}
\hline & \multicolumn{4}{|c|}{ PRE TEST } & \multicolumn{3}{|c|}{ POST TEST } & \multirow[b]{2}{*}{$\mathrm{T}$} \\
\hline & Grado & N. ${ }^{\circ}$ & Media & Percentil & $\mathrm{T}$ & Media & Percentil & \\
\hline Agrupam. & $3^{\circ}$ prim. & 4 & 22.5 & 35 & 48.3 & 19.25 & 25 & 43.3 \\
\hline \multirow[t]{3}{*}{ Primaria } & $4^{\circ}$ prim. & 4 & 13.75 & 33.75 & 45.7 & 12.25 & 31.25 & 41.6 \\
\hline & $5^{\circ}$ prim. & 5 & 7 & 20 & 37.58 & 8.6 & 34 & 44.12 \\
\hline & & & 14.41 & 29.58 & 43.86 & 13.36 & 30.08 & 43 \\
\hline Agrupam. & $1^{\circ} \mathrm{sec}$ & 7 & 12 & 30 & 42.12 & 10.71 & 22.14 & 38.07 \\
\hline \multirow[t]{2}{*}{ Secundaria } & $2^{\circ} \mathrm{sec}$ & 3 & 16.66 & 31.66 & 42.3 & 20 & 41.66 & 46.6 \\
\hline & & & 14.33 & 30.83 & 42.21 & 15.35 & 31.9 & 42.33 \\
\hline
\end{tabular}

Tal como predecíamos, el nivel de Comprensión Lectora de los alumnos con problemas de aprendizaje expuestos al Programa Experimental se vio incrementado en mayor medida que el nivel de Comprensión Lectora de los alumnos con problemas de aprendizaje no sometidos al Programa Experimental. Pero ello ocurrió fundamentalmente en el Agrupamiento II (1. ${ }^{\circ}$ y $2{ }^{\circ}$ de secundaria). Los alumnos del Agrupamiento I también mostraron progresos, pero en menor grado, algo que más adelante analizaremos y discutiremos. 
Para visualizar de mejor modo lo que estamos señalando, veamos el siguiente cuadro y el gráfico correspondiente:

Cuadro N. ${ }^{\circ}$ 3. Comparación de medias en CLP de los sujetos del grupo experimental pre y pos test por agrupamientos.

\begin{tabular}{ccc}
\hline Grupo Experimental & Pre Test & Post Test \\
\hline Agrupamiento I & 11.2 & 12.19 \\
Agrupamiento II & 11.7 & 14.41 \\
\hline
\end{tabular}

Gráfico $\mathbf{N} \bullet^{\circ}$ 1. Comparación de medias en grupo experimental por agrupamiento.

También se evaluó cómo había sido este progreso en comprensión lectora (hipótesis de trabajo), en base al Programa de intervención, diferenciados por grados escolares y por niveles de lectura (Cuadro N. ${ }^{\circ}$ ).

Cuadro N. ${ }^{\circ}$ 4. Comparición de medias en CLP de los sujetos del grupo experimental pre y post test por niveles de lectura (Agrupamiento I, primaria).

\begin{tabular}{cccccccccc}
\hline \multicolumn{7}{c}{ Medias de grupo experimental en CLP } \\
\hline Grado & $\begin{array}{c}\text { Nivel de } \\
\text { lectura }\end{array}$ & \multicolumn{2}{c}{ Pre test } & Post test & Gl & T \\
\hline & & N. $^{0}$ & Media & Percentil & Media & Percentil & & \\
$3^{\text {o }}$ Prim. & Segundo nivel & 3 & 15.33 & 20 & 17.66 & 36.6 & & \\
$4^{\text {o }}$ Prim. & Tercer nivel & 6 & 10.83 & 16.6 & 12.16 & 33.3 & & \\
$5^{\text {o }}$ Prim. & Cuarto nivel & 4 & 7.5 & 17.5 & 6.75 & 15 & & & \\
& & & 11.2 & & 12.19 & & 12 & $\mathrm{t}=0.685$ & $\begin{array}{c}\text { No hay } \\
\text { dif. }\end{array}$ \\
\hline
\end{tabular}




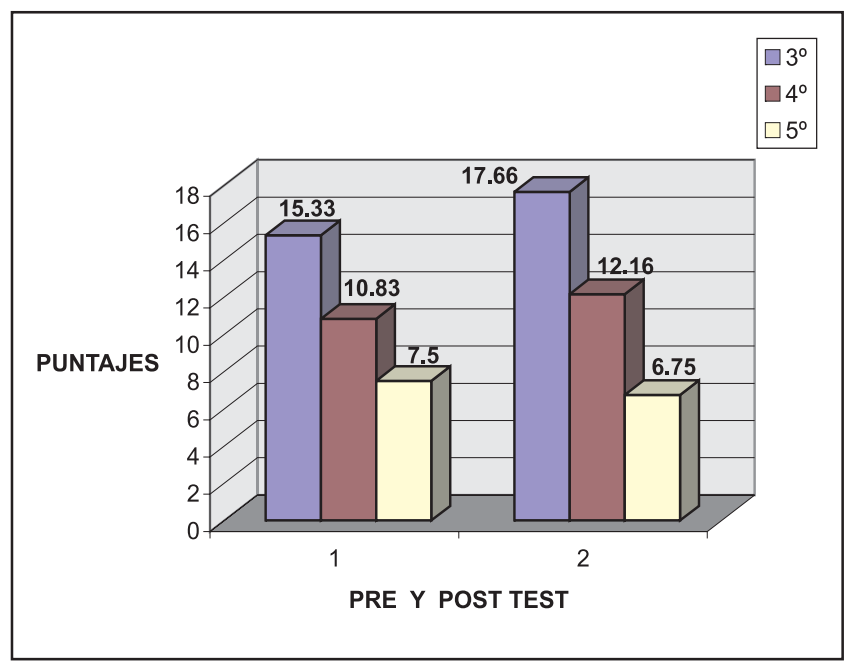

Gráfico N. ${ }^{\circ}$ 2. Medias en CLP-grupo experimental por grados primaria.

Cuadro N. ${ }^{\circ}$ 5. Comparación de medias en CLP de los sujetos del grupo experimental pre y post test por niveles de lectura (Agrupamiento II, secundaria).

\begin{tabular}{|c|c|c|c|c|c|c|c|c|c|}
\hline GRADO & $\begin{array}{l}\text { NIVEL DE } \\
\text { LECTURA }\end{array}$ & & $\begin{array}{l}\text { PRE } \\
\text { TEST }\end{array}$ & & $\begin{array}{l}\text { POST } \\
\text { TEST }\end{array}$ & & GL & $\mathrm{t}$ & \\
\hline & & $\mathrm{N}^{0}$ & Media & Percentil & Media & Percentil & & & \\
\hline $1^{\circ}$ secundaria & Sexto Nivel & 4 & 8.25 & 15 & 10 & 22.5 & & & \\
\hline \multirow[t]{2}{*}{$2^{\circ}$ secundaria } & Séptimo Nivel & 6 & 15.16 & 26 & 18.83 & 35 & & & \\
\hline & & & 11.7 & & 14.41 & & 9 & $\mathrm{t}=2.225$ & *Hay dif. Sign. \\
\hline
\end{tabular}

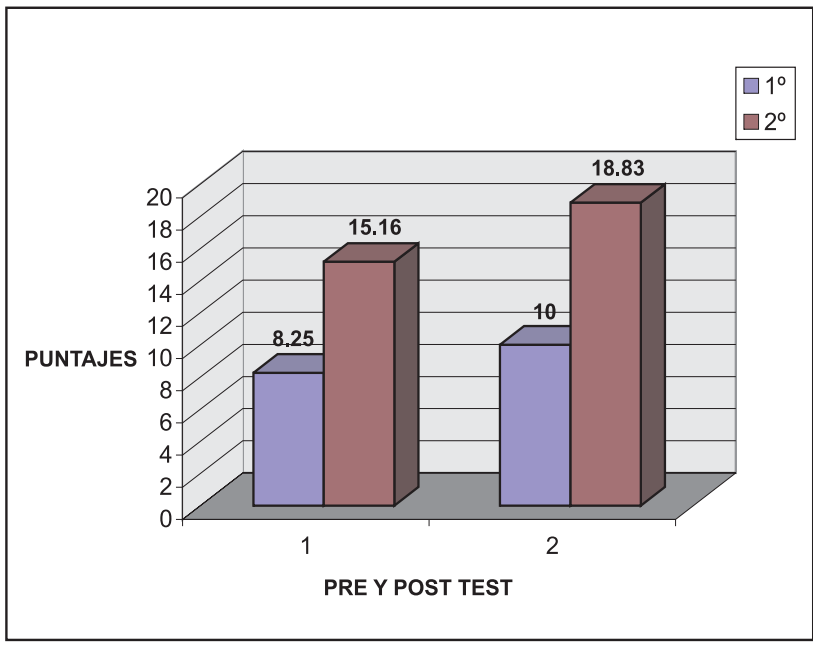

Gráfico $\mathbf{N} .^{\circ}$ 3. Comparación de medias en CLP grupo experimental secundaria. 
En el estudio se encontró que el conjunto de los alumnos del grupo experimental mostró avances importantes, a excepción del $5{ }^{\circ}$ grado de primaria.

Los alumnos de $3 .^{\circ}$ de primaria avanzaron de un percentil 20 a percentil 36 , lo cual indica que mejoraron su habilidad para comprender textos simples (algunas pocas oraciones relacionadas entre sí). Debemos indicar que una media de 17.6 y percentil 36 equivale a una puntuación $\mathrm{T}$ de 44.9 , lo cual quiere decir que los sujetos se ubican a 5.1 por debajo del promedio.

Los alumnos de $4 .^{\circ}$ de primaria avanzaron de un percentil 17.7 a percentil 34.4 , lo cual indica que dieron un avance importante en su habilidad para comprender textos simples, sobre todo, en la captación del sentido del párrafo, así como en su capacidad inferencial para la comprensión de textos con variedad de situaciones y personajes. Sin embargo, también debemos indicar que una media de 12.16 y percentil 34.4 equivale a una puntuación $\mathrm{T}$ de 41.85 , lo cual quiere decir que los sujetos se ubican a 8.15 por debajo del promedio (cerca de una desviación típica).

Los alumnos de $5 .^{\circ}$ de primaria decrecieron levemente de un percentil 17.5 a percentil 15, el mismo que equivale a una puntuación $\mathrm{T}$ de 38.92 , lo cual indica que son alumnos con problemas serios para globalizar la información, distinguir entre hechos y opiniones, categorizar y establecer relaciones causales entre los hechos, aspectos que como se verá tienen mucho que ver con el pensamiento verbal. Asimismo, una media de 6.75 y percentil 15 equivale a una puntuación $\mathrm{T}$ de 38.92 , es decir los sujetos se ubican a 11.08 puntos por debajo del promedio.

En el caso de los alumnos de secundaria, tenemos lo siguiente:

- Los alumnos de $1 .^{\circ}$ de secundaria avanzaron de un percentil 15 a percentil 22.5 , lo cual indica que tuvieron un avance importante en su habilidad para señalar en forma clara las relaciones significativas existentes entre los elementos de textos narrativos con hechos claramente estructurados y con sujetos individuales y colectivos concretos. Sin embargo, también, se debe indicar que un percentil 22.5 y una media de 10 equivale a una puntuación $\mathrm{T}$ de 38.92 , lo cual quiere decir que los sujetos se ubican a 11.08 por debajo del promedio.

- Los alumnos de $2 .^{\circ}$ de secundaria avanzaron de un percentil 26.6 a percentil 35 , lo cual indica que tuvieron un avance importante en su habilidad para captar el sentido preciso dentro del texto, situar hechos en el plano temporal y espacial correctos, y realizar inferencias adecuadas. El percentil de 35 que alcanzan y una media de 18.83 equivale a una puntuación $\mathrm{T}$ de 44.93 , lo cual quiere decir que los sujetos se ubican a 5.07 por debajo del promedio.

En conclusión, el conjunto de los sujetos del Grupo Experimental, agrupados por grados escolares y niveles de lectura, logró avances importantes, luego de ser expuestos al Programa Experimental.

Respecto a las hipótesis secundarias, se halló que no existe relación entre las habilidades sintácticas-semánticas y la comprensión lectora en los niños con problemas de aprendizaje de la muestra válida (46 sujetos), pero, interesantemente, si se halló una relación de $\mathbf{0 . 4 9}$, 
que, por el tamaño de la muestras, es significativo al nivel del 0,05 entre dichas habilidades sintácticas-semánticas y la comprensión lectora en los niños con problemas de aprendizaje luego de haber sido expuestos al Programa Experimental (Cuadro N. ${ }^{\circ}$ ).

Cuadro N. ${ }^{\circ}$ 6. Matriz de correlación entre Cloze y CLP (grupo experimental post test).

\begin{tabular}{llc}
\hline & CLOZE & CLP \\
\hline CLOZE & 1.000 & \\
CLP & $\mathbf{0 . 4 9 8}$ & 1.000 \\
& & \\
\hline \multicolumn{2}{l}{ NUMBER OF OBSERVATIONS: } & 23 \\
\hline
\end{tabular}

En el estudio se observó que la relación hallada en la muestra experimental entre habilidades sintácticas y semánticas y la comprensión lectora, al concluirse el programa experimental, fue más significativa.

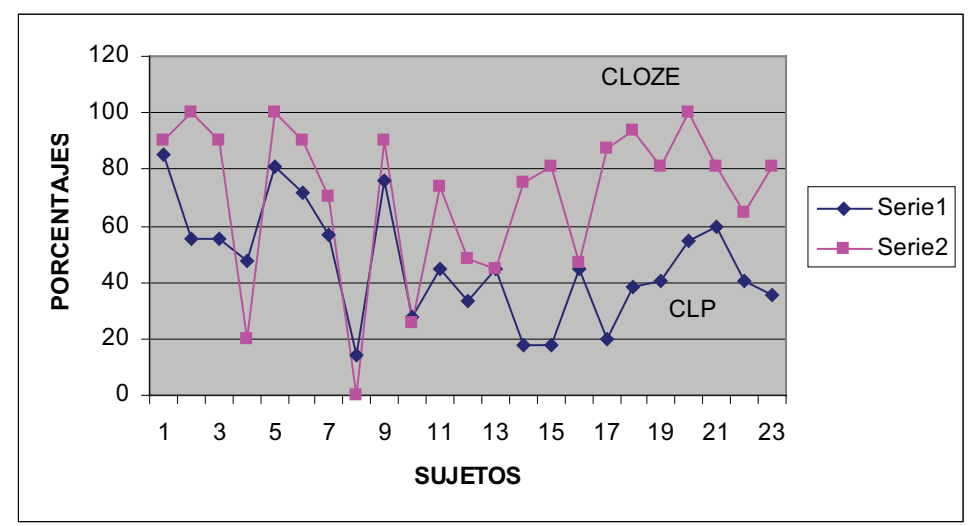

Gráfico N. ${ }^{\circ}$ 4. Correlación entre Cloze y CLP (grupo experimental post test).

Los otros aspectos a evaluar fueron la relación entre inteligencia verbal y comprensión lectora, así como entre memoria verbal de corto plazo y comprensión lectora. Sobre lo primero, se halló una relación moderada: 0.261, la cual es significativa al nivel del 0,05 en muestra de 42 sujetos (muestra total válida) (Cuadro N. ${ }^{\circ}$ ). 
Cuadro N. ${ }^{\circ}$ 7. Matriz de correlación entre CIV y CLP (muestra válida pre test).

\begin{tabular}{lll}
\hline & CIV & CLP \\
\hline CIV & 1.000 & \\
CLP & 0.261 & 1.000 \\
& & \\
\hline NUMBER OF OBSERVATIONS: & 46 \\
\hline
\end{tabular}

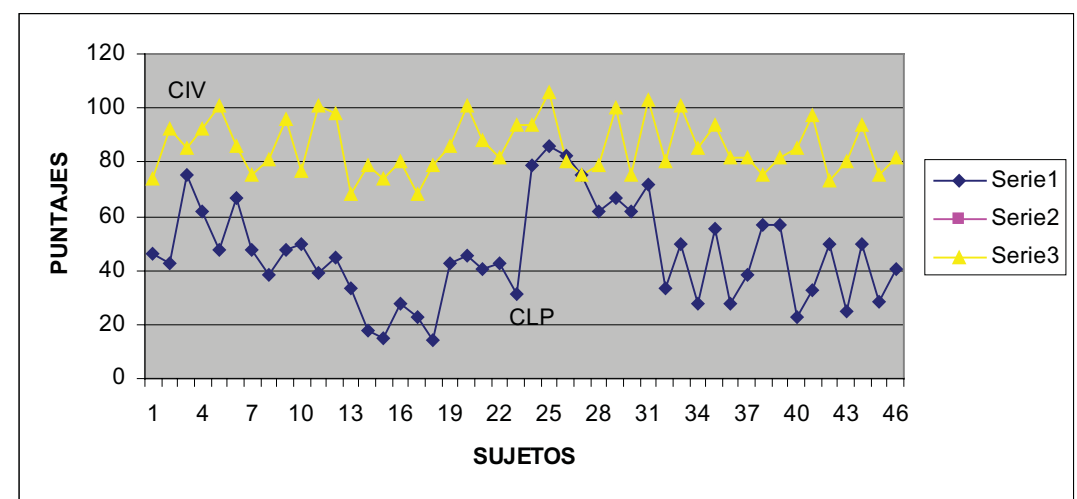

Gráfico N. ${ }^{\circ}$ 5. Correlación entre CIV y CLP (muestra total).

Respecto a la relación entre memoria verbal de corto plazo y la comprensión lectora en la muestra válida, no se halló relación.

\section{DISCUSIÓN}

Como se esperaba, los sujetos con problemas de aprendizaje, diferenciados por grados y agrupamientos (primarios y secundarios), sometidos al Programa Experimental, mostraron incremento importante en su rendimiento en comprensión lectora, entre el pre y el post test. En este sentido, se considera que existe aquí un patrón definido respecto a la relación entre Programas de Intervención y adquisición de Habilidades de Comprensión Lectora, en este caso, en alumnos con problemas de aprendizaje.

Como bien sabemos, los estudios sobre la lectura -aspecto crucial en el desarrollo cognitivo humano- son recientes en nuestro medio. La propia investigación psicolingüística, contemporánea a nivel internacional, sigue ahondando en el tema de la lectura comprensiva. Aquí, por supuesto, una de las más importantes contribuciones viene por el lado teórico de la vertiente trabajada por Carpenter y Just (1987) y Van Dijk y Kintsch (1983), quienes han señalado que la comprensión viene a ser producto de la construcción de un modelo 
mental y un modelo situacional que busca encontrar el significado de lo que aparece en el texto, articulando lo que se expresa en el mismo con los conocimientos y experiencias previas del sujeto.

Más adelante, Kintsch $(1988,1991,1992,1998)$ afinó su modelo de 1983 y formuló el modelo Construcción-Integración (CI). Uno de los puntos principales de reajuste y desarrollo en la teoría de Kintsch tiene que ver con la diferencia crucial entre lo que proporciona el texto en sí (y que luego el sujeto lector estructura en su mente a manera de red de proposiciones) y lo que el propio sujeto aporta (importantísimo papel de los conocimientos previos).

Por otro lado, es importante resaltar el trabajo que, en el campo investigativo sobre la comprensión lectora y, en particular, sobre la importancia del diseño de Programas de Intervención, viene realizando un grupo de psicólogos españoles, encabezados por García Madruga, J., Luque J.L., y Sánchez E., quienes destacan precisamente aquella relación crucial entre Programas de intervención y mejoramiento de la comprensión lectora.

Los resultados hallados en nuestro estudio, precisamente, guardan coherencia con lo encontrado por los investigadores mencionados. En una investigación reciente (García Madruga, Elosúa, Luque, Gárate, 1999), se presenta con claridad la relación directa entre un Programa de Intervención y la habilidad para construir la Macroestructura de los textos. Trabajando con 45 sujetos como grupo experimental, diferenciados en dos niveles: EGB (27 ss) y BUP (18 ss), dichos autores encontraron que en los tres tipos de tareas planteadas: construcción de macroestructura, aprendizaje y uso de macrorreglas, y recuerdo macroestructural, la diferencia fue clara a favor del grupo experimental.

Ahora, quizá lo particular de nuestro aporte radique en el hecho de haber comprobado empíricamente que dichas mejoras pueden realizarse incluso en alumnos con problemas de aprendizaje.

\section{Importancia del factor evolutivo}

En relación al hallazgo principal mencionado, es importante ahora analizar, interpretar y discutir las diferencias encontradas al compararse el rendimiento del grupo experimental, en términos de Agrupamiento I (alumnos del $3 .^{\circ}$ al $5 .^{\circ}$ de primaria) y alumnos del Agrupamiento II $\left(1 .^{\circ}\right.$ y $2 .^{\circ}$ de secundaria).

Se ha visto que el grupo de alumnos pertenecientes al Agrupamiento II fue el que obtuvo los progresos más significativos, comparados a los alumnos del Agrupamiento I, cuyo progreso fuer positivo también, pero en menor grado.

Esta diferencia en el proceso de mejora de la comprensión lectora se explicaría, además de los efectos en sí del Programa, por un factor evolutivo. Los sujetos del Agrupamiento II, que correspondían a alumnos de $1 .^{\circ}$ y $2 .^{\circ}$ de secundaria, cuya edad promedio era 13.3 años, mostraron progresos más significativos que los alumnos del Agrupamiento I, cuya edad promedio era 9.8 años, probablemente debido a que sus mejores niveles de atención y memoria (que aumentan con la edad, según Pascual Leone, 1980, Kail, 1986, Case $1974,1985)$ repercutirían en la mejora de su rendimiento frente a las tareas de activación 
de sus conocimientos previos, identificar y reconocer las ideas principales, además de su mejora cualitativa en los procesos básicos que tienen que ver con la decodificación y el reconocimiento de palabras.

\section{La importancia de ir mejorando el aprendizaje de estrategias}

Respecto a las diferencias halladas en el rendimiento de los alumnos grado por grado y en relación con los niveles de lectura alcanzados, habría que señalar que en general mostraron un avance importante, a excepción del grupo de $5 .{ }^{\circ}$ de primaria. Ello se debe a que en dicho grupo se encontraban los alumnos con mayor trastorno del aprendizaje (disléxicos, retardo lector severo), quienes, frente a las tareas de mayor exigencia y complejidad planteada, no respondieron positivamente al tratamiento. Tal como lo señalaba Bravo (1996), trabajar con niños disléxicos y/o niños que presentan retardo lector severo es muy difícil. Por lo general, tan sólo en el mediano plazo (tratamientos de 1 a 2 años) se puede apreciar algunos signos importantes de avance. Por lo demás, a medida que los alumnos van avanzando de grado debe ponerse mayor énfasis en la enseñanza de estrategias para la comprensión de textos, superando el simple nivel de la decodificación de palabras.

\section{La relación entre habilidades sintácticas-semánticas y la comprensión lectora mejora con el Programa de Intervención}

De acuerdo al modelo de la arquitectura funcional, acerca de la lectura, sabemos que en los procesos de decodificación lectora son determinantes los llamados procesos de bajo nivel, así como los de nivel medio. Dentro de los primeros se encuentran básicamente los procesos perceptivos: movimientos oculares de fijación y saccádicos, análisis visual de rasgos y de letras, fundamentalmente. Y dentro de los procesos de nivel medio, están los procesos léxicos, es decir de reconocimiento de palabras. Pero, para demostrar competencia en la lectura comprensiva, se requiere poner en marcha, además, procesos de alto nivel: procesos sintácticos y semánticos, así como memoria operativa y procesos inferenciales.

Dado que en dicha tarea compleja se deben realizar labores de abstracción, categorización, análisis de la coherencia local del texto, aplicación de inferencias, construcción de la representación mental del texto, etc., entonces se hace necesario contar con dichas habilidades a nivel mental, pero sobre la base de lo primero. Lo que nuestro primer resultado empírico, en esta área, estaría indicando es que en la muestra de alumnos con problemas de aprendizaje, el rendimiento en la prueba CLOZE, inicialmente, estaría muy influenciado por las severas deficiencias que dichos alumnos tenían en la decodificación, en la escritura y en la comprensión. Frente a ello, muchos de los niños habrían echado mano a factores "naturales" como Inteligencia, nivel de activación y predisposición para este tipo de tareas, etc. en busca de compensar los déficits antes mencionados, mostrándose, por tanto, una casi inexistente relación entre dichas habilidades de alto nivel y la comprensión lectora en un primer momento.

Pero en relación al segundo resultado empírico, en términos del efecto que pudo haber provocado el Programa de Intervención, a nivel de las habilidades sintácticas y semánticas, 
incrementándolas a la par que mejoraban las destrezas decodificadoras y de comprensión lectora, hay que señalar que más allá de dicho dato lo interesante radicaría en reflexionar acerca de la importancia de dichas habilidades de base, sintácticas y semánticas, y cómo pueden mejorar las mismas.

En la última reformulación de su modelo teórico (Kintsch 1974, 1988, 1993, 1994, Kintsch y Van Dijk, 1978, Van Dijk y Kintsch, 1983), Kintsch plantea un punto de vista sumamente interesante respecto a la forma en que a nivel mental se construiría la representación de un texto, cuando estamos tratando de comprenderlo. En lo fundamental, lo que se plantea es que cuando intentamos comprender un texto, no es que tratemos de elaborar una copia literal del mismo (precisamente las personas con menor nivel de comprensión lectora, son las que se limitan a la reproducción literal) sino que tratamos de captar la esencia semántica del mismo, esto quiere decir captar, asimilar y recordar las ideas principales. De aquí se desprende que cuando intentamos abstraer un texto, debemos ir más allá de lo que las palabras o la frase en sí están diciendo, para avanzar a captar o interpretar o construir el significado global del mismo.

En nuestro estudio, a partir de la relación hallada, a nivel de post test, entre dichas habilidades sintácticas y semánticas y las competencias lectoras, se ratificaría dicha formulación teórica. Asimismo, de alguna manera, quedó demostrada también que dichas habilidades pueden ser mejoradas con el entrenamiento.

\section{Acerca de la inteligencia verbal y la memoria verbal de corto plazo}

En el estudio, se evidencia una importante relación entre Inteligencia verbal y comprensión lectora. Este resultado es muy importante. Si partimos de la idea central de que comprender realmente un texto significa ir más allá del nivel literal, y avanzar hacia la interpretación global de lo que el autor ha querido decir (captar las proposiciones centrales, es decir construir la macroestructura), entonces nos daremos cuenta de lo importante que vienen a ser en dicha habilidad compleja los procesos cognitivos de alto nivel, y entre ellos, sin lugar a dudas, estarán las funciones de abstracción y de inferencia lógica.

García Madruga (1987) investigó, en lengua española, el papel central de las inferencias, planteando que sin ellas sería prácticamente imposible que el sujeto comprenda los textos en forma global. Es la inferencia, proceso cognitivo de alto nivel, la que permite que el sujeto vaya articulando el sentido esencial de las oraciones a nivel de una estructura global coherente.

De acuerdo a nuestro estudio, la correlación hallada entre Cociente de Inteligencia Verbal y Comprensión Lectora guarda enorme importancia, más aún si tomamos en cuenta la correlación de mayor significación entre el subtest de Semejanzas Lógicas y Comprensión. Ahí, estaría actuando aquella importante habilidad cognitiva inferencial a la que hemos hecho referencia líneas arriba.

En relación a la memoria verbal, habría que señalar que la inexistente correlación hallada en la muestra, se debe a que solamente estábamos evaluando a nivel de memoria, a la memoria verbal de corto plazo (subtest "Memoria de Dígitos" en el WISC-R), y que la 
Memoria Operativa, tal como lo entiende Baddeley $(1981,1983,1986)$, aparte de cumplir con las funciones de almacenamiento de corto plazo, también cumpliría un rol fundamental en el control y procesamiento activo de la información, constituyéndose de este modo en el proceso cognitivo central en los complejos asuntos del procesamiento de textos.

\section{Acerca del programa de tratamiento experimental dirigido a mejorar la comprensión lectora}

Respecto al programa de tratamiento empleado, lo primero que se debe señalar es que demostró sus bondades al haber mejorado - de modo importante, si bien no de modo altamente significativo, debido a que se trataba de una muestra de niños y adolescentes con graves problemas de aprendizaje de la lectura- en la muestra experimental de 23 sujetos, sus niveles de comprensión lectora.

El presente estudio ha permitido atender de modo particular la forma en que estarían funcionando en el sujeto lector las importantes habilidades sintácticas, semánticas, la memoria verbal de corto plazo y las inferencias, al parecer decisivas para la comprensión lectora; así como, observar, investigar y tratar las importantes estrategias psicolingüísticas particulares de supresión, generalización y elaboración, que son fundamentales en la comprensión de textos. Se considera que, interviniendo en estos procesos cognitivos y psicolingüísticos, el sujeto mejoraría su rendimiento en la comprensión lectora, hipótesis que luego fue confirmada.

\section{CONCLUSIONES}

1. Luego de realizar el estudio con los 23 sujetos de la muestra experimental, comparados con los 23 alumnos del grupo control, en función a nuestro Objetivo general e Hipótesis principal, se halló diferencia significativa entre los niveles de Comprensión Lectora alcanzados en el pre test y el post test, por los alumnos con problemas de aprendizaje del Agrupamiento de secundaria, expuestos al Programa Experimental, así como una mejoría importante en los alumnos del Agrupamiento de primaria.

2. En términos generales, los alumnos sometidos al Programa de Tratamiento mostraron mejoría en su rendimiento a nivel de comprensión lectora, y en particular los alumnos de $3 .^{\circ}$ de primaria y los de $2 .^{\circ}$ de secundaria, se aproximaron a la media $\mathrm{T}$ (50 puntos). Sin embargo, los niveles de logro no fueron plenamente satisfactorios, entre otras razones, debido a que se trataba de alumnos con problemas serios de aprendizaje en la lectura.

\section{Objetivos específicos e Hipótesis secundarias}

1. Respecto a la probable relación entre habilidades sintácticas-semánticas y la comprensión lectora (pre test: muestra total), se encontró que no existe relación entre dichas variables. 
2. Dicha relación si quedó demostrada en el grupo experimental (post test), encontrándose que los procesos de alto nivel, procesos sintácticos-semánticos, pueden ser mejorados y son determinantes en la comprensión lectora.

3. Respecto a la relación entre Inteligencia verbal y Comprensión lectora, se confirma la importancia que, para la lectura comprensiva, tienen las funciones de abstracción, generalización y de formulación de inferencias, todas ellas integrantes de la inteligencia verbal o lingüística.

4. Respecto a la ausencia de relación entre Memoria verbal de corto plazo y la Comprensión lectora, se debe continuar con el estudio de las variables de Memoria Operativa y Comprensión de Textos.

5. Respecto a si se darían cambios significativos a nivel de habilidades sintácticassemánticas entre pre test y post test, hay que señalar que se produjo un relativo progreso en el grupo experimental comparado con el grupo control.

6. Acerca de los Instrumentos, la prueba CLP y la prueba y técnica CLOZE demostraron su utilidad y validez, en tanto permitieron acceder a dimensiones cognitivas y psicolingüísticas de la comprensión lectora.

El Programa Experimental diseñado expresamente para el presente estudio fue muy útil, pues se alcanzó los objetivos trazados en la presente investigación.

\section{REFERENCIAS BIBLIOGRÁFICAS}

1. Baddeley, A. D. (1999). “Working memory”. En: García M., J. et al. Comprensión lectora y memoria operativa. Barcelona: Edit. Paidós.

2. Ministerio de Educación del Perú (2001). Boletín CRECER N. ${ }^{\circ}$ 9. Lima: Edic. MINEDU,

3. Bravo, L. (1995). Lenguaje y dislexia. Enfoque cognitivo del retardo lector. Santiago de Chile: Edit. Universidad Católica de Chile.

4. _ (1991). Psicología de las dificultades del aprendizaje escolar. Santiago de Chile: Edit. Universitaria.

5. Bravo, L.; Bermeosolo y Pinto (1987). "Dislexia inicial: Predictividad de su evolución”. Anales de la Facultad de Educación 10. Santiago de Chile: Universidad Católica de Chile.

6. Canales, R. (1997). Actitudes de la madre y desarrollo psicolinguistico en niños de zona urbano marginal del Callao: Un estudio sobre cultura, afectividad y lenguaje. Tesis de Maestría en Psicología. Lima: Universidad Nacional Mayor de San Marcos.

7. Carpenter, P. y Just, M.(1987). "The psychology of reading and language comprensión”. En: García M., J. Martin C., Luque y Santamaría (1995). Comprensión y adquisición de conocimientos a partir de textos. Madrid: Siglo Veintiuno. 
8. Claux, M. L. y La Rosa (2004). La comprensión de lectura en el aula: una experiencia significativa. Lima: Pontificia Universidad Católica.

9. Clemente, M. y Domínguez, A. (1999). La enseñanza de la lectura. Enfoque psicolinguÍstico y sociocultural. Madrid: Pirámide.

10. Condemarín, M. y Milicic, N. (1988). Test de Cloze. Santiago de Chile: Edit. Andrés Bello.

11. Condemarín, M., Alliende, F. y Milicic, N. (1990). Prueba CLP. Formas Paralelas. Santiago de Chile: Universidad Católica de Chile.

12. Cuetos, F. (1996). Psicología de la lectura. Barcelona: Praxis.

13. De Vega, M.; Carreiras, M.; Gutiérrez-Calvo M. y Alonso-Quecuty M.L. (1990). Lectura y comprensión: una perspectiva cognitiva. Madrid: Alianza Editorial.

14. García Madruga J.A.; Martín Cordero, J.I., Luque; J.L. y Santamaría (1995). Comprensión y adquisición de conocimientos a partir de textos. Madrid: Siglo Veintiuno.

15. García Madruga, J.A.; Gárate, M.; Elosúa, M.R.; Luque, J.L. y Gutiérrez, F. (1999). Comprensión lectora y memoria operativa. Barcelona: Paidós.

16. Gonzáles, R. (1998). "Comprensión lectora en estudiantes universitarios iniciales". "Persona". Revista de la Facultad de Psicología N. ${ }^{\circ}$ 1. Lima: Fondo de Desarrollo Editorial, Universidad de Lima.

17. Jiménez, J. y Ortiz, M. (1995). Conciencia fonológica y aprendizaje de la lectura. Teoría, evaluación e intervención. Madrid: Síntesis.

18. Molina, S.; Sinués, A.; Deaño, M.; Puyuelo, M.; Bruna, O. (1998). El fracaso en el aprendizaje escolar (II). Dificultades específicas de tipo neuropsicológico. Málaga: Ediciones Aljibe.

19. Noriega, G. (1997). Estudio cualitativo de los niveles de comprensión de un grupo de niños deficientes y buenos lectores antes y después de un Programa de Intervención. Tesis para optar el grado de Magíster en Psicología. Lima: Universidad Nacional Mayor de San Marcos.

20. Pinzás, J. (1997). Metacognición y lectura. Lima: Edic. Pontificia Universidad Católica del Perú.

21. Tapia, V. (1996). "Desarrollo de un programa de lectura en niños deficientes lectores". En: Psicología cognitiva y sus aplicaciones a la clínica educacional. II Seminario Internacional. Lima: Escuela Profesional de Psicología, Universidad San Martín de Porres.

22. Valle F. y Cuetos, F. (1989). "Las dislexias desde el enfoque neurocognitivo". Revista de Neurología de Sant Pau. Madrid.

23. Vallés, A. (1995). Técnicas de velocidad y comprensión lectora. Madrid: Edit. Escuela Española. 
24. Van Dijk y Kintsch (1978). "Toward a model of text comprensión and production". Psychological Review 85 y En: García M., J. et al. (1999). Comprensión lectora y memoria operativa. Barcelona: Paidós.

25. Velarde, E. (2001). Relación entre la conciencia fonológica y el nivel de decodificación y comprensión lectora en niños de 8 años del $3^{\circ}$ grado de primaria de dos niveles socioeconómicos del Cercado del Callao. Tesis de Maestría en Problemas de Aprendizaje. Lima: UNIFE. 